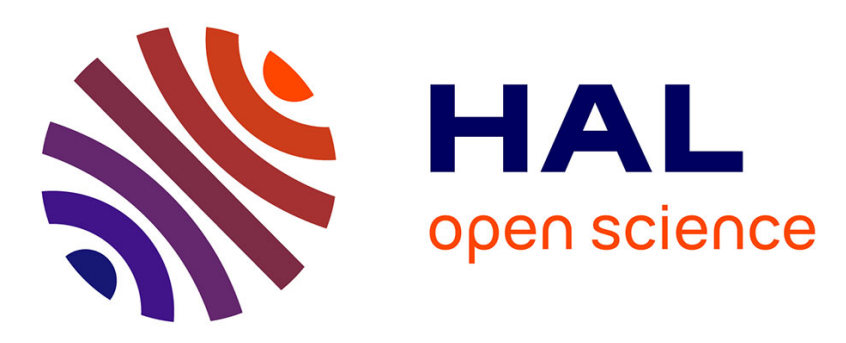

\title{
Prediction of Ventricular Mechanics After Pulmonary Valve Replacement in Tetralogy of Fallot by Biomechanical Modeling: A Step Towards Precision Healthcare
}

Maria Gusseva, Tarique Hussain, Camille Hancock Friesen, Gerald Greil, Dominique Chapelle, Radomir Chabiniok

\section{To cite this version:}

Maria Gusseva, Tarique Hussain, Camille Hancock Friesen, Gerald Greil, Dominique Chapelle, et al.. Prediction of Ventricular Mechanics After Pulmonary Valve Replacement in Tetralogy of Fallot by Biomechanical Modeling: A Step Towards Precision Healthcare. Annals of Biomedical Engineering, 2021, 49 (12), pp.3339-3348. 10.1007/s10439-021-02895-9 . hal-03462902

\author{
HAL Id: hal-03462902 \\ https://hal.science/hal-03462902
}

Submitted on 2 Dec 2021

HAL is a multi-disciplinary open access archive for the deposit and dissemination of scientific research documents, whether they are published or not. The documents may come from teaching and research institutions in France or abroad, or from public or private research centers.
L'archive ouverte pluridisciplinaire HAL, est destinée au dépôt et à la diffusion de documents scientifiques de niveau recherche, publiés ou non, émanant des établissements d'enseignement et de recherche français ou étrangers, des laboratoires publics ou privés. 


\section{Title page}

\section{Title}

Prediction of ventricular mechanics after pulmonary valve replacement in tetralogy of

Fallot by biomechanical modeling: A step towards precision healthcare

\section{Authors}

Maria Gusseva ${ }^{1,2}$

Tarique Hussain ${ }^{3}$

Camille Hancock Friesen 4

Gerald Greil ${ }^{3}$

Dominique Chapelle ${ }^{1,2}$

Radomír Chabiniok ${ }^{1,2,3,5}$

\section{Affiliations}

\section{${ }^{1}$ Inria, Palaiseau, France}

'LMS, Ecole Polytechnique, CNRS, Institut Polytechnique de Paris, Palaiseau, France ${ }^{3}$ Division of Pediatric Cardiology, Department of Pediatrics, UT Southwestern Medical Center, Dallas, TX

${ }^{4}$ Division of Pediatric Cardiothoracic Surgery, Department of Surgery, University of Nebraska, Medical Center College of Medicine, Omaha, NE

${ }^{5}$ Department of Mathematics, Faculty of Nuclear Sciences and Physical Engineering, Czech Technical University in Prague, Czech Republic

\section{Running Head}

Prediction of RV mechanics after pulmonary valve replacement

\section{Authors' contribution:}

- Conception of the study: T.H. D.C., R.C.

- Statistical analysis: M.G.

- Models formulation: D.C., R.C.

- Analyze and interpretation of the results: M.G., T.H., G.G., C.H.F., R.C.

- Design of the figures: M.G.

- Drafting manuscript: M.G., R.C.

- Editing and revising manuscript: M.G., T.H., C.H.F., G.G., D.C., R.C.

- Approving final version of manuscript: M.G., T.H., C.H.F., G.G., D.C., R.C.

\section{Corresponding Author}

Radomír Chabiniok, MD, PhD

Address: Department of Pediatrics, UT Southwestern Medical Center, 5323 Harry Hines Blvd, Dallas, TX 75390, USA

ORCID ID: 0000-0002-7527-2751

Tel: +12144567311

E-mail: radomir.chabiniok@UTSouthwestern.edu 


\section{Abstract and key terms}

\section{Abstract}

Clinical indicators of heart function are often limited in their ability to accurately evaluate the current mechanical state of the myocardium. Biomechanical modeling has been shown to be a promising tool in addition to clinical indicators. By providing a patient-specific measure of myocardial active stress (contractility), biomechanical modeling can enhance the precision of the description of patient's pathophysiology at any given point in time. In this work we aim to explore the ability of biomechanical modeling to predict the response of ventricular mechanics to the progressively decreasing afterload in repaired tetralogy of Fallot (rTOF) patients undergoing pulmonary valve replacement (PVR) for significant residual right ventricular outflow tract obstruction (RVOTO). We used nineteen patient-specific models of patients with rTOF prior to pulmonary valve replacement (PVR), denoted as $P S M_{\text {pre, }}$ and patientspecific models of the same patients created post-PVR $\left(P S M_{\text {post }}\right)$ - both created in our previous published work. Using the $P S M_{\text {pre }}$ and assuming cessation of the pulmonary regurgitation and a progressive decrease of RVOT resistance, we built relationships between the contractility and RVOT resistance post-PVR. The predictive value of such in silico obtained relationships were tested against the $P S M_{\text {post }}$, i.e. the models created from the actual post-PVR datasets. Our results show a linear 1-dimensional relationship between the in silico predicted contractility post-PVR and the RVOT resistance. The predicted contractility was close to the contractility in the $P S M_{\text {post }}$ model with a mean ( \pm SD) difference of $6.5( \pm 3.0) \%$. The relationships between the contractility predicted by in silico PVR vs. RVOT resistance have a potential to inform clinicians about hypothetical mechanical response of the ventricle based on the degree of pre-operative RVOTO. 
Keywords: myocardial contractility, ventricular overload, valvular heart disease, biomechanical modeling, valve replacement 


\section{Introduction}

After initial repair of tetralogy of Fallot (TOF) pulmonary regurgitation (PR) and/or residual right ventricular outflow tract obstruction (RVOTO) detrimentally affect ventricular mechanical properties in the long-term. A chronic right ventricular (RV) volume and/or pressure overload leads to RV dilatation and/or hypertrophy. At the extent of the myocardial ability to compensate, the $\mathrm{RV}$ remodeling become pathologic and RV systolic and/or diastolic function may deteriorate. To avoid decompensated RV remodeling or to allow the RV to reverse-remodel back to normal size, patients undergo a pulmonary valve replacement (PVR). Timing of PVR is critical. PVR is ideally performed as late as possible because of the limited lifespan of an implanted valve $^{32}$, but before the myocardium undergoes significant irreversible changes. The ability to most accurately evaluate the current mechanical state of the myocardium and subsequently predict optimal timing of PVR is a subject of ongoing research. The sensitivity and specificity of the current clinical guidelines for PVR are limited and in practice post-PVR RV reverse-remodeling occurs in only $60 \%$ of patients ${ }^{33}$. The level of PR, RV dilatation (end-diastolic volume typically indexed to body surface area), and $\mathrm{RV}$ ejection fraction are often considered as the main clinical indicators for $P V R^{3,13}$. A recent observational cohort study has demonstrated that greater RV hypertrophy and higher levels of RV systolic pressures are strongly associated with irreversible remodeling and/or sudden death in tetralogy of Fallot patients after surgical repair $(\mathrm{rTOF})^{41}$. It is therefore important to understand both the independent and combined effect of RV dilation and hypertrophy triggering factors (PR and RVOTO, respectively) on the evolution of RV mechanics.

Biomechanical modeling has been shown to be a promising tool for modeling "in silico" (a new term that has been coined to contrast to "in vivo") myocardial function. 
The short-term models (capturing single or a few cardiac beats) have been shown to be useful in predicting short-term effects of Cardiac Resynchronization Therapy²8,31,38. Biomechanical modeling has also been proposed to assess the effect of pulmonary vasodilatation therapy in Fontan patients with early-stage heart failure ${ }^{35}$.

In our recently published retrospective study ${ }^{15}$ we demonstrated the ability of a biomechanical model to provide information about the current mechanical state of the myocardium in late rTOF patients prior to and immediately after PVR, while using the clinical data obtained pre- and post-PVR. In the present work we would like to explore the ability of these patient-specific models created from the pre-PVR data $\left(P S M_{p r e}\right)$ to predict the response of ventricular mechanics to PVR. Specifically, we aim to build a relationship between the myocardial contractility predicted in silico using $P S M$ pre, while decreasing the RVOT resistance (and subsequently decreasing RV-to-PA pressure difference, in clinical medicine often incorrectly called the pressure gradient). This will simulate the reduction of RVOTO during PVR when the valve is implanted. The predictive capability of such an in silico PVR will be assessed by comparing the predicted RV contractility with the contractility in the patient-specific models post-PVR $\left(P S M_{\text {post }}\right)$ as reported in our previous work $^{15}$, where $P S M_{\text {post }}$ and the associated contractility values were obtained when using the catheterization data measured after the actual PVR.

\section{Materials and Methods}

\subsection{Patient-specific biomechanical models pre- and post-PVR}

The creation of $P S M_{\text {pre }}$ and $P S M_{\text {post }}$ of the studied cohort has been reported previously ${ }^{15}$. Here, we provide only a brief description. We used a biomechanical model of the RV cavity with simplified geometry (spherical ventricle with inner radius $R$ 
and wall thickness $d$ ). The model developed by Caruel et al. ${ }^{5}$ has been previously employed in various clinical studies ${ }^{24,36}$. The constitutive mechanical properties of the myocardium are preserved as in the full 3D heart model ${ }^{9}$.

The overall tissue-level mechanical behavior of the myocardium is described by a rheological model of Hill-Maxwell type ${ }^{17}$ that allows to represent an active contractile component (actin-myosin unit) and a visco-elastic component (collagen-rich elements). The active behavior of the myocardium is modeled within A.F. Huxley's sliding filament theory ${ }^{20,22}$. The myosin filaments within a sarcomere unit are considered as an element that produces active stress $\left(\tau_{c}\right)$ and active stiffness $\left(k_{c}\right)$ at certain sarcomere extension $\left(e_{f i b}\right)$ with the following system of differential equations ${ }^{4,9}$ :

$$
\left\{\begin{array}{l}
\dot{k}_{c}=-\left(|u|+\alpha\left|\dot{e}_{f i b}\right|\right) k_{c}+n_{0}\left(e_{f i b}\right) k_{0}|u|_{+} \\
\dot{\tau}_{c}=-\left(|u|+\alpha\left|\dot{e}_{f i b}\right|\right) \tau_{c}+\dot{e}_{f i b} k_{c}+n_{0}\left(e_{f i b}\right) \sigma_{0}|u|_{+}
\end{array}\right.
$$

where $u(t)$ is an electrical activation function that is related to intracellular calcium kinetics and induces contraction (when $u>0$ ) or relaxation (when $u<0$ ) of the myocardium. The parameter $\alpha$ is a bridge destruction rate upon rapid change in length in the sarcomere ${ }^{9}$. The function $n_{0}\left(e_{f i b}\right) \in[0,1]$ is the representation of the FrankStarling law that gives the fraction of recruited myosin heads at a given sarcomere extension $\left(e_{f i b}\right)^{9}$. The parameters $\sigma_{0}$ and $k_{0}$ are the active stress and stiffness developed by the sarcomere during systole under optimal fiber extension $\left(e_{f i b}\right)$, when $n_{0}=1$, reflecting the inotropic state ${ }^{36}$. The parameter $\sigma_{0}$ will be further referred to as contractility. The elastic part of passive behavior of the myocardium is modeled by the constitutive law inspired by Holzapfel and $O g d e n^{16,18}$ in the form of the hyperelastic potential:

$$
W_{e}=C_{0} e^{C_{1}\left(J_{1}-3\right)^{2}}+C_{2} e^{C_{3}\left(J_{4}-1\right)^{2}}
$$


where $C_{i}, i \in\{0,1,2,3\}$ are material constants that account for the passive behavior of the ventricle under imposed preload pressure. The calibration of $C_{i}$ to the given enddiastolic pressure (EDP) and volume provides the patient-specific end-diastolic pressure volume relationship (EDPVR). The overall dynamics of the system are given by a Lagrangian formulation, where $\underline{\underline{e}}$ is Green-Lagrange strain tensor and $J_{1,4}$ are the reduced invariants of the right Cauchy-Green strain tensor $(\underline{\underline{C}})$, that accounts for transversely isotropic behavior of the myocardium (for details see ${ }^{18,9,5}$ ).

The viscous part of the passive behavior is given by viscous pseudo-potential:

$$
W_{v}=\frac{\eta}{2} \operatorname{tr}\left(\underline{\underline{\dot{e}}}^{2}\right)
$$

where $\eta$ is a viscous damping parameter.

The ventricular cavity model was coupled with inlet and outlet valves to control the inflow from the tricuspid valve (TV) and outflow through the right ventricular outflow tract (RVOT). Both TV and RVOT were represented by a system of diodes with forward and backward resistances that allowed simulation of the required forward and backward flows in the corresponding compartments (Figure 1). Thus, by increasing the resistance to forward flow ( $R_{\text {for }}^{T V}$ and $R_{\text {for }}^{R V O T}$ for TV and RVOT, respectively) we can simulate valvular stenosis of a given grade. Likewise, by decreasing the backward resistances ( $R_{\text {back }}^{T V}$ and $R_{\text {back }}^{R V O T}$ for TV and RVOT, respectively) we can model valvular regurgitation. The pressures in the atrium, ventricle and pulmonary artery $\left(P_{a t}, P_{R V}\right.$ and $P_{P A}$, respectively) are coupled with a ventricular volume change $\dot{V}$ (inflow or outflow of blood) as described by Sainte-Marie et al. ${ }^{37}$ 


$$
\begin{cases}\dot{V}=\frac{\left(P_{a t}-P_{R V}\right)}{R_{\text {for }}^{T V}}+\frac{\left(P_{P A}-P_{R V}\right)}{R_{\text {back }}^{R V O T}}, & \text { when } P_{R V} \leq P_{a t} \\ \dot{V}=\frac{\left(P_{R V}-P_{a t}\right)}{R_{\text {back }}^{T V}}+\frac{\left(P_{P A}-P_{R V}\right)}{R_{\text {back }}^{R V O T}}, & \text { when } P_{a t} \leq P_{R V} \leq P_{P A} \\ \dot{V}=\frac{\left(P_{P A}-P_{R V}\right)}{R_{\text {for }}^{R V O T}}+\frac{\left(P_{a t}-P_{R V}\right)}{R_{\text {back }}^{T V}}, & \text { when } P_{R V} \geq P_{P A}\end{cases}
$$

The circulation system is represented by a two-stage Windkessel model ${ }^{39}$, as is also depicted in Figure 1.

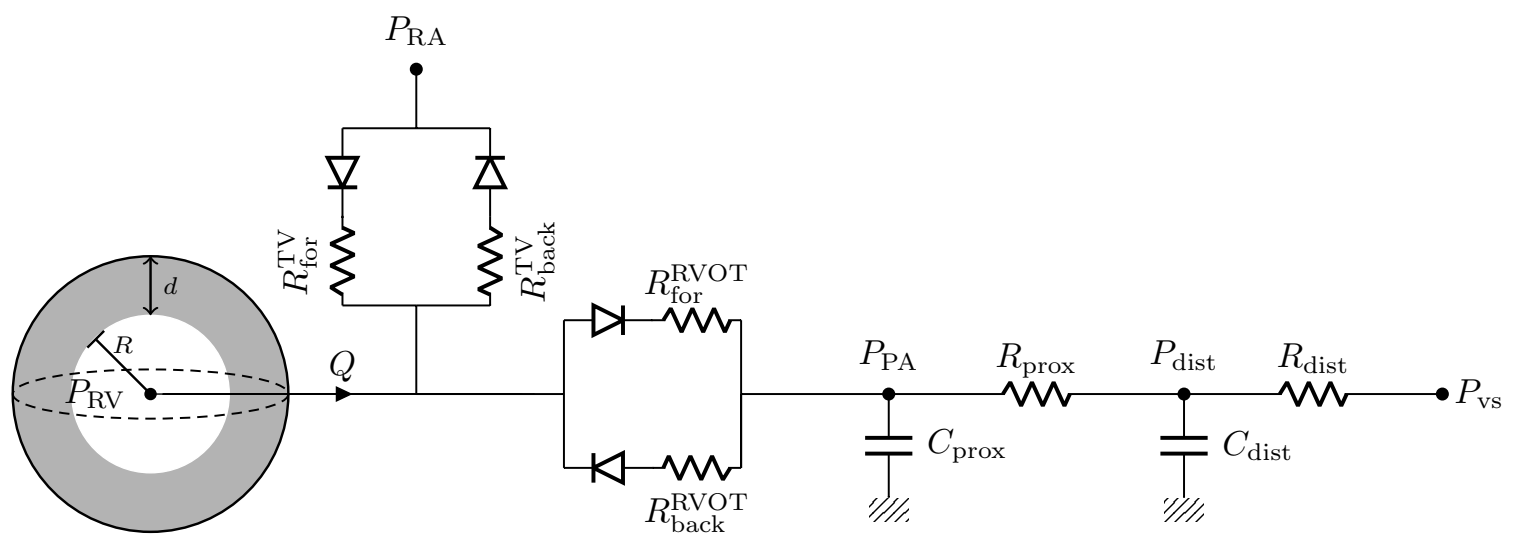

Figure 1. Reduced-order model of the right ventricle (RV) with inner radius $R$ and wall thickness $d$. The ventricle is coupled to tricuspid valve (TV) and RV outflow tract (RVOT) via a system of diodes with forward and backward resistance $\left(R_{\text {for }}^{T V}, R_{\text {back }}^{T V}\right.$ and $R_{\text {for }}^{R V O T}, R_{\text {back }}^{R V O T}$, respectively). The pulmonary circulation is represented by a Windkessel model with resistance and capacitance of proximal and distal arteries $\left(R_{\text {prox }}, C_{\text {prox }}\right.$, and $R_{\text {dist }}, C_{\text {dist }}$, respectively). $P_{R V}$ and $P_{R A}$ are pressure in the RV and right atria, respectively. $P_{P A}$ and $P_{d i s t}$, and $P_{v S}$ are pressure in the proximal and distal pulmonary artery, and venous system, respectively.

In the present study, we will use $P S M_{\text {pre }}$ and $P S M_{\text {post }}$ of nineteen rTOF patients created in our previous work ${ }^{15}$. These models are given by the patient-specific parameters characterizing the circulation (proximal and distal resistance and capacitances $R_{p}, R_{d}, C_{d}$ ); the heart geometry and function (myocardial passive stiffness, active contractility), and RVOT mechanics (RVOT forward and backward 
resistances $\left.R_{\text {for }}^{R V O T}, R_{\text {back }}^{R V O T}\right)$. The detailed model calibrations are described in our previous work ${ }^{15}$. We remark that the ethical approvals in the study ${ }^{15}$, in which the patient-specific models were created, were granted by the Institutional Review Boards of UT Southwestern Medical Center Dallas (STU-2020-0023) and UT Austin (IRB 2020-06-0128).

\subsection{Post-PVR in silico prediction of contractility}

The pre-PVR patient-specific models $P S M_{\text {pre }}$ were used to predict in silico the RV contractility after PVR. We assumed no PR after PVR (therefore we increased the backward RVOT resistance $R_{\text {back }}^{R V O T}$ to a large value). Sequentially, the RVOT resistance $R_{\text {for }}^{R V O T}$ was decreased to its minimum (representing no RVOTO assumed after PVR). For each value of $R_{f o r}^{R V O T}$ the ventricular contractility was recalibrated in the model to preserve pulmonary artery peak systolic pressure (PA PSP). This is in line with our observations made in this cohort ${ }^{15}$ and another study using clinical data ${ }^{29}$ and is detailed in Section 3.1. The RVOT pressure difference for the corresponding decrease of the $R_{\text {for }}^{R V O T}$ resistance was calculated by subtracting PA PSP from the simulated RV pressure. The in silico decrease of RVOT pressure difference was plotted against the corresponding values of in silico predicted contractility. The contractility in $P S M_{\text {post }}$ reported previously ${ }^{15}$ (see Section 2.1) was used to validate the in silico predicted contractility for a measured value of RVOT pressure difference post-PVR. 


\subsection{Statistical analysis}

The changes in post-PVR RV and PA PSP were evaluated via the Wilcoxon signedrank test at $p<0.050$. The difference between the in silico predicted post-PVR contractility and the contractility in $P S M_{\text {post }}$ was quantitatively evaluated via a BlandAltman plot analysis. In addition, the in silico predicted contractility and the contractility in $P S M_{\text {post }}$ were multiplied by the ratio of myocardial wall thickness over ventricular chamber radius - rescaled in accordance with the Laplace law. Pearson's correlations were built between the rescaled in silico predicted contractility, rescaled contractility in $P S M_{\text {post }}$ and the post-PVR RV peak systolic pressure (RV PSP) at $p<0.050$.

\section{Results}

3.1. RV and PA peak systolic pressures in patient-specific models pre- and post-PVR

Patients were divided into 2 groups according to the level of RVOTO: low- and highRVOTO groups based on RV-to-LV systolic pressure ratio. RVOTO was defined as low if the ratio of $\mathrm{RV}$ to $\mathrm{LV}$ peak systolic pressures was $<50 \%$ and high if the ratio was $\geq 50 \%$. All patients from the low-RVOTO group had at least moderate PR (regurgitation fraction $>30 \%$ ), while the high-RVOTO patients had at least mild PR (regurgitation fraction $>20 \%$ ). There were $n=9$ patients with low-RVOTO and $n=10$ patients with highRVOTO. Complete clinical and hemodynamic characteristics of this patient cohort can be found in the supplementary material of published work ${ }^{15}$.

Figure 2 shows RV PSP and PA PSP pre- and post-PVR in the low- and highRVOTO groups. RV PSP showed insignificant post-PVR change $(p=0.133)$ and a 
significant decrease $(p=0.004)$ in low-RVOTO group and high-RVOTO group, respectively. In the low-RVOTO and high-RVOTO groups the median change of PA PSP was 2.0 and $1.0 \mathrm{mmHg}$, respectively. The analysis of pressure data in our patient cohort therefore showed an insignificant change of PA PSP post-PVR $(p=0.326$ for all patients).
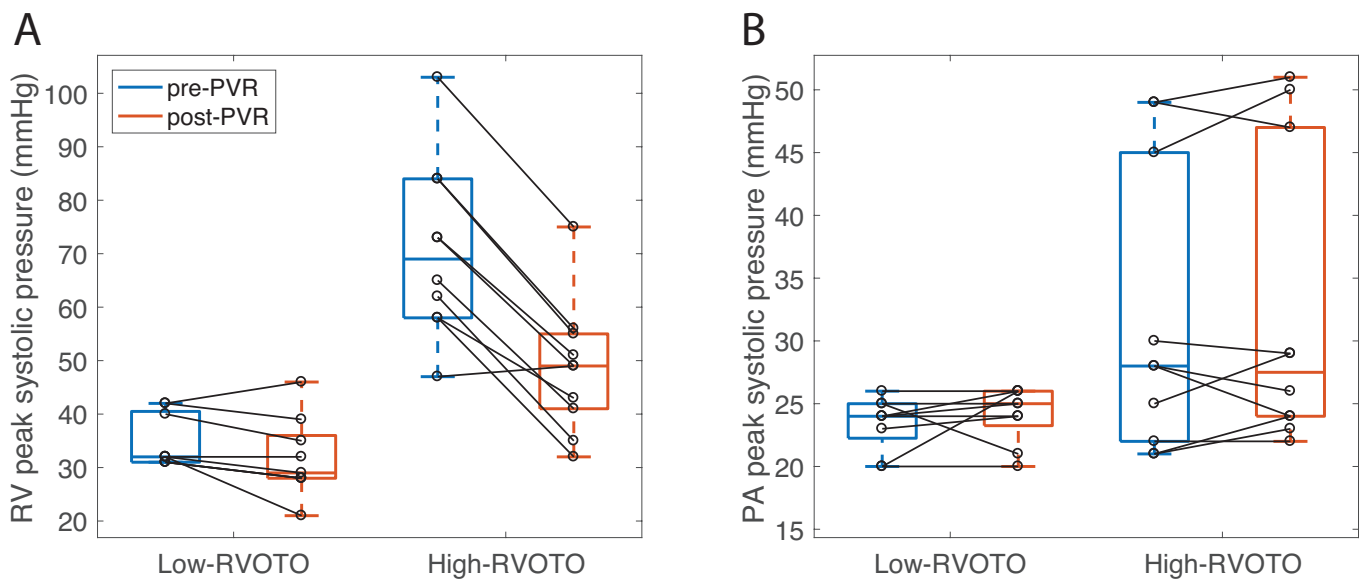

Figure 2. A: right ventricular (RV) peak systolic pressure pre- and post-PVR. B: pulmonary artery (PA) peak systolic pressure pre- and post-PVR. Central line inside the boxplots is the median, bottom and top edges of the boxes show 25th and 75th percentiles, respectively, the whiskers connect the most extreme data points not considered as outliers. Low-/High- RVOTO: low/high right ventricular outflow tract obstruction patient groups.

\subsection{In silico prediction of right ventricular contractility}

Figures 3 and 4 show the in silico prediction of RV contractility for a sequential decrease of RVOT resistance $\left(R_{f o r}^{R V T}\right)$ in 4 patients from high- and 2 patients from lowRVOTO groups, respectively. In silico predictions of contractility for the remaining 
patients can be found in the Supplementary Figures S1 and S2. With a decreasing level of $R_{f o r}^{R V O T}$, the RV contractility was decreasing along a line for all studied patients, creating a 1D patient-specific trajectory within the RV contractility-RVOT pressure difference space. The mean $( \pm \mathrm{SD})$ in silico predicted contractility at $0 \mathrm{mmHg}$ RVOT pressure difference for all patients was $36( \pm) 9 \mathrm{kPa}$. Note that the range of healthy RV contractility was assumed to be $38-48 \mathrm{kPa}^{15}$.

Figure $5 \mathrm{~A}$ shows a Bland-Altman plot of the difference between the in silico predicted contractility post-PVR and data-estimated contractility in $P S M_{\text {post }}$ for the measured RVOT pressure difference post-PVR.

The mean $( \pm S D)$ absolute difference between the in silico predicted contractility and the contractility in $P S M_{\text {post }}$ in the low- and high-RVOTO group was $4.0( \pm 2.6) \mathrm{kPa}$ and $3.1( \pm 2.1) \mathrm{kPa}$, respectively. Pearson's correlation $\left(R^{2}\right)$ for the in silico predicted contractility and for the contractility in PSM post (both multiplied by the ratio of myocardial wall thickness over ventricular chamber radius according to Laplace's law) was 0.902 $(p=0.000)$ and $0.910(p=0.000)$, respectively (Figure 5B). Figure 5C shows Pearson's correlation for the in silico predicted contractility at $0 \mathrm{mmHg}$ pressure difference and the contractility in pre- and post-PVR patient-specific models rescaled in accordance with the Laplace's law in relation to the RV PSP. $R^{2}$ for all values of contractility (orange, and blue points and black stars in Figure $5 \mathrm{C})$ is $0.954(p=0.000)$. For the contractility at $0 \mathrm{mmHg}$ pressure difference alone (black stars in Figure $5 \mathrm{C}$ ), $R^{2}$ is 0.850 $(p=0.000)$. 

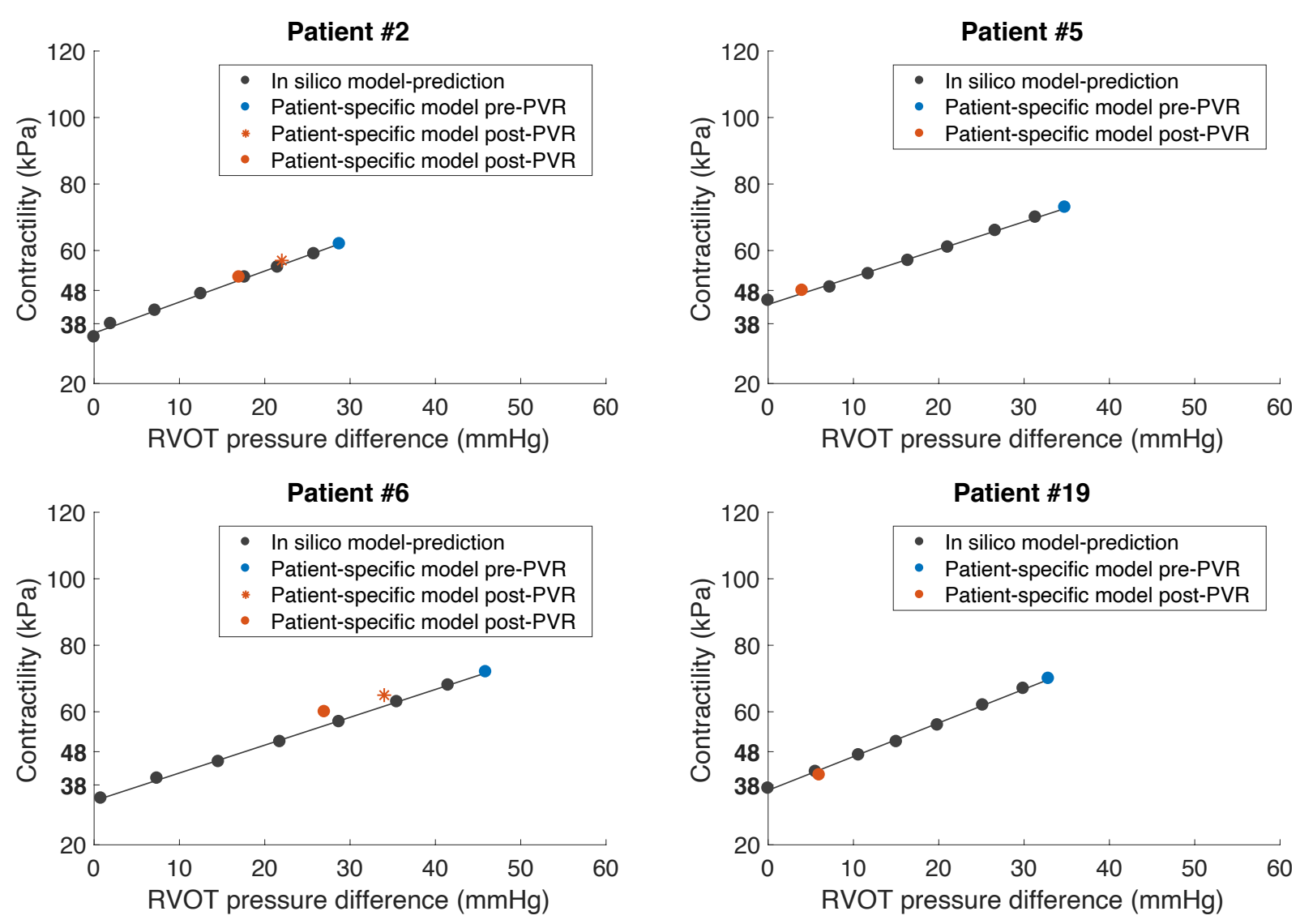

Figure 3. In silico prediction of right ventricular (RV) contractility post-PVR (black points) for selected patients from high right ventricular outflow tract obstruction (RVOTO) group. Filled blue and orange points are the contractilities in the pre- and post-PVR patient-specific models. Orange stars in patients \#2 and \#6 are additional post-PVR data-estimated contractility, where post-PVR pressures were acquired $\sim 2$ hours earlier than the final post-PVR pressure measurement (filled orange points). Healthy $\mathrm{RV}$ contractility was assumed to be $38-48 \mathrm{kPa}^{15}$. 

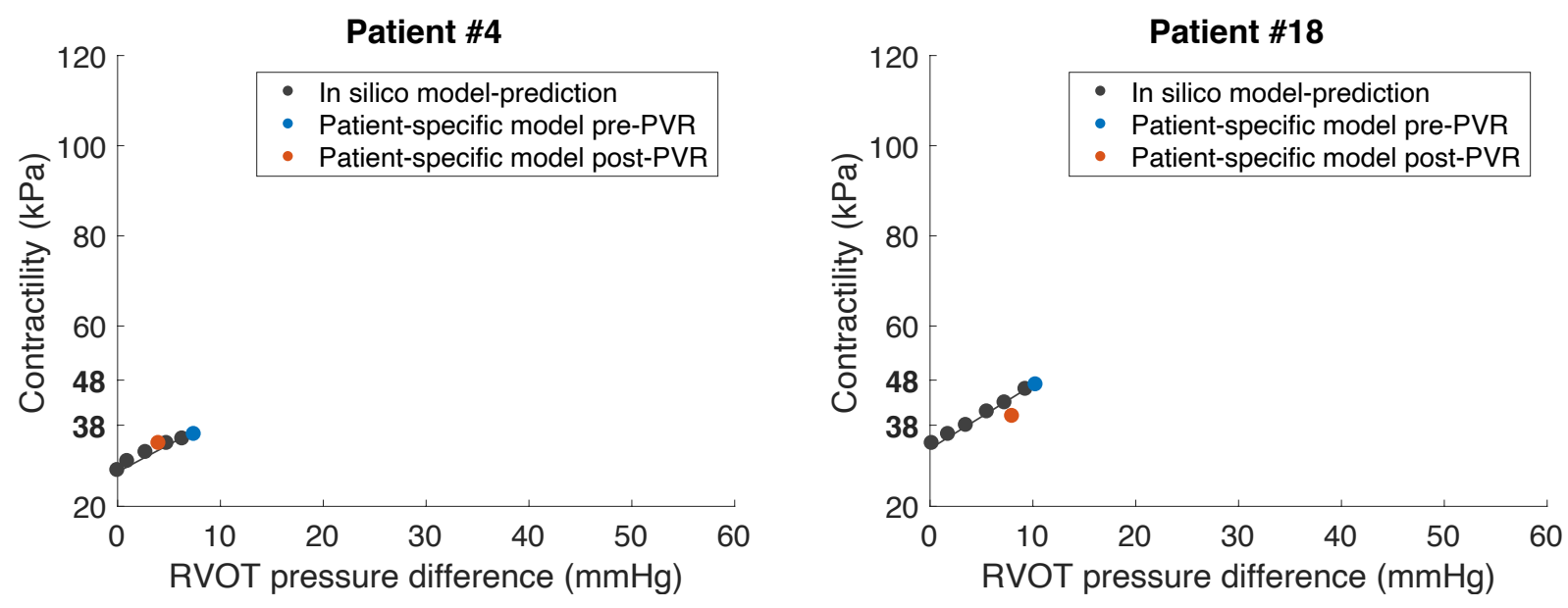

Figure 4. In silico prediction of right ventricular (RV) contractility post-PVR (black points) for selected patients from low right ventricular outflow tract obstruction (RVOTO) group. Filled blue and orange points are the contractilities in the pre- and post-PVR patient-specific models.
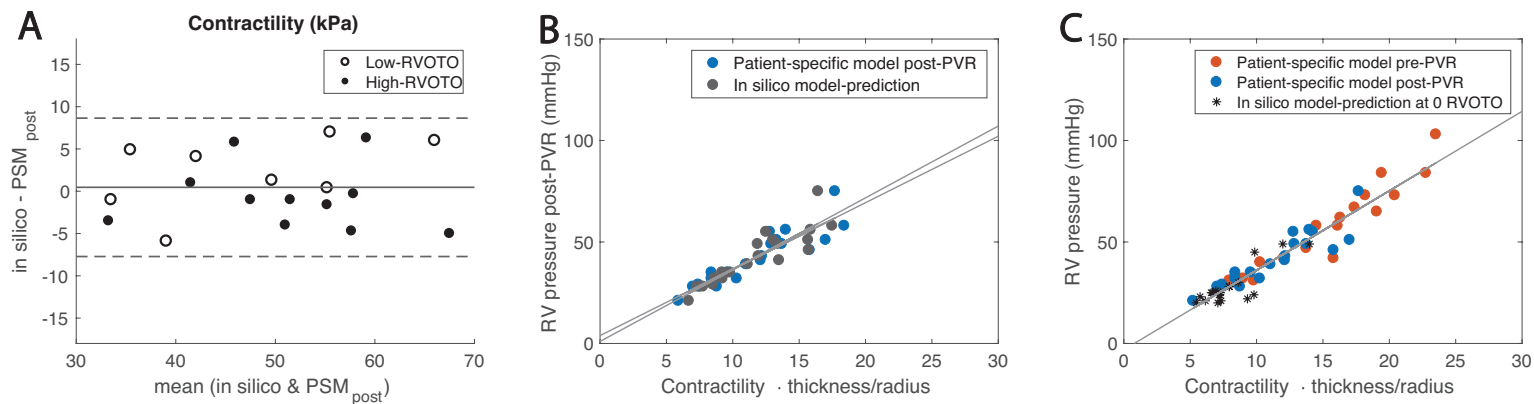

Figure 5. Quantitative difference between the contractility from in silico prediction and that from the post-PVR patient-specific models for a measured post-PVR right ventricular (RV) outflow tract pressure difference. A: Bland-Altman plot, solid horizontal line is the mean of the difference between the in silico predicted and contractility in the patient-specific model, top and bottom dashed horizontal lines are the limits of agreement at $95 \%$ prediction interval ( \pm 1.96 times standard deviation). B: Pearson's correlation for in silico predicted and the contractility in patient-specific model, while both are multiplied by the ratio of myocardial wall thickness over ventricular chamber radius (rescaled contractility) and measured RV peak systolic pressure post-PVR at $p<0.05$. C: Pearson's correlation between the rescaled contractility and RV pressure. Orange and blue points are the pre- and post-PVR contractilities in the patient-specific models, respectively. Black stars are the in silico predicted contractility at zero mmHg RVOT pressure difference, $R^{2}=0.954, p=0.000$. 


\section{Discussion}

In this work we evaluated the predictive value of patient-specific biomechanical models created from patients' data prior to PVR in previously reported study ${ }^{15}$ to establish model-predicted (in silico) changes in RV contractility post-PVR. We used an assumption that PA PSP was preserved post-PVR as it showed little or no variation in clinical data ${ }^{15,29}$. We therefore gradually decreased the RVOT resistance and recalibrated the contractility to maintain PA PSP for each patient to represent the set of in silico predicted contractilities. We used the data-estimated contractility in $P S M_{\text {post }}$, also created in the previous study ${ }^{15}$, to validate the in silico predicted contractility. For the majority of patients, the in silico predicted contractility fell close to the RV contractility in $P S M_{\text {post. }}$ The mean $( \pm S D)$ error in low- and high-RVOTO groups corresponded to $5.0( \pm 4.0) \%$ and $4.0( \pm 3.0) \%$ with respect to pre-PVR contractility, respectively. The difference between the in silico predicted contractility and the contractility in $P S M_{\text {post }}$ on individual patient basis varied from negative (negative part of $y$-axis on Bland-Altman plot denoting the in silico predictions underestimating the contractility in $P S M_{\text {post }}$ ) to positive (positive part of y-axis, the in silico predictions overestimating the contractility from patient-specific models). These errors could be explained by the measurement uncertainties in the catherization pressure signals. In addition, different preload pressures imposed during the in silico PVR prediction vs. the preload used in the patient-specific models could have contributed to the discrepancy presented in the Bland-Altman plot: the in silico prediction did not assume any preload change from the pre-PVR RV end-diastolic pressure (EDP), while the actual post-PVR patient-specific models directly used the measured post-PVR pressures. However, as only a mild decrease of EDP post-PVR was observed, we 
expect that its effect on the post-PVR contractility will be small compared to that given by the decrease in RVOTO. We also evaluated the difference between the in silico predicted contractility values and the contractility in $P S M_{\text {post }}$ rescaled with respect to the Laplace's law (Wall stress directly proportional to Pressure * chamber radius / wall thickness). Pearson's correlations between the rescaled contractility and measured RV pressure post-PVR - using either the in silico prediction or the contractility in $P S M_{\text {post }}$ - showed almost identical strong correlations (Figure 5B). This suggests that the effect of the variation present in Bland-Altman plot is small with respect to the global mechanical properties of the ventricle for a given ventricular geometry.

We observe linearity of the relationship between the in silico predicted contractility and the RVOT resistance. In addition, these 1D relationships predict the contractility at $0 \mathrm{mmHg}$ RVOT pressure difference to be in the range of healthy $\mathrm{RV}$ contractility ${ }^{15}$ in all patients. In accordance with Laplace's law we plotted the rescaled in silico predicted contractility at $0 \mathrm{mmHg}$ RVOT pressure difference against the prePVR PA PSP and combined that correlation with previously published Laplace's correlations of pre- and post-PVR data-estimated contractility in $P S M_{\text {pre }}$ and $P S M_{\text {post }}$, respectively ${ }^{15}$. The combined correlation revealed a strong overall relationship. The return to a completely unobstructed RVOT may not be possible in some patients due to the residual RVOT gradient of at least $10-15 \mathrm{mmHg}$ (as a result of placing a stented bioprosthesis). Nevertheless, the in silico predicted contractility at hypothetically minimal RVOT resistance also follows the Laplace's law of ventricular wall stress generation. This further strengthens the physiological relevance of the in silico predicted relationship between the contractility vs. RVOT resistance post-PVR for a given ventricular geometry. 
Although the model used in this study is rather complex and nonlinear in various aspects (e.g. passive and active behavior of myocardium), the final finding is a nearly linear relationship between the in silico predicted contractility and the RVOT resistance throughout a wide range of the resistance values. As shown in Figure 2, there is an insignificant change of PA PSP post-PVR. With the systolic PA pressure waveform being rather flat and PA PSP being close to PA end-systolic pressure (ESP), the pressure change between the start and end of ejection is nearly preserved post-PVR as well as the amount of ejected blood (stroke volume, SV). This was also observed in the clinical study of Lurz et al. ${ }^{29}$, which suggested that the SV undergoes insignificant change immediately after PVR.

We can approximate SV by

$$
S V=\frac{R V P S P-P A P S P}{R_{\text {for }}^{R V T}} * t_{\text {eject }},
$$

where $t_{\text {eject }}$ stands for time of ejection. In the previous work ${ }^{15}$ we demonstrated that model-derived contractility $\sigma_{0}$ is proportional to RV PSP via the Laplace law:

$$
R V P S P=2 \sigma_{0} * \frac{h}{r}
$$

where $h$ and $r$ are ventricular wall thickness and chamber radius, respectively. Assuming that SV is relatively unchanged immediately after the RVOTO release, by combining Eqs. (5) and (6) the linearity between $\sigma_{0}$ and RVOT resistance $\left(R_{\text {for }}^{R V O T}\right)$ becomes apparent: 


$$
2 \sigma_{0} * \frac{h}{r}=\frac{S V * R_{f o r}^{R V O T}}{t_{\text {eject }}}+P A P S P
$$

We hypothesize that the linearity between the in silico predicted contractility and the RVOT resistance observed in the current cohort of patients is determined by the preservation of SV immediately post-PVR. A larger cohort of patients containing both RVOTO and PR should be studied to elucidate if the preservation of the SV post-PVR and if the observed linearity consistently happens. Nevertheless, the contractility itself accounts for nonlinear active constitutive behavior of the myocardium and in the present modeling approach is an inherently patient-specific index of the inotropic state of the myocardium and our results support its prognostic significance.

It should be recognized that there are other mechanical models that could provide the representation of the internal myocardial stress. For example, a lumpedparameter model of the heart and circulation as in CircAdapt is an alternative patientspecific approach for modeling the stress-strain relationship of the myocardium and the interaction between ventricles and the vascular tree ${ }^{1,26,27}$. Even though CircAdapt models contain the interaction between the two ventricles and are fast in computation time (real time or close to real time), their micromechanical formulation does not account for sarcomere-level kinetics but is in the form of phenomenological myofiberlevel stress-strain relationship. The family of 3D finite element models ${ }^{30,19,9,31}$ allows the investigation of regional wall mechanics (e.g. contractility ${ }^{7,2}$ ), see also review articles $^{8,42}$ and references therein. However, these are computationally expensive approaches, which limits their practical applicability in the investigation of large cohorts of patients in clinical settings. In the current work we ran 7 simulations per patient to establish the in silico relationships. This would be hardly achievable with a 3D model. 
Overall, the in silico predicted relationship between the contractility and RVOT resistance demonstrates an immediate response of $\mathrm{RV}$ active mechanical properties to the varying afterload pressure. Our results suggest that after PVR the RVOT pressure difference and myocardial contractility will follow a nearly linear relationship (1D line). The linearity of pressure vs. contractility relationship could be used to inform about evolution of RV mechanics at a given level of RVOTO post-PVR and subsequently assist in planning the patient's intervention. Should the goal be to reduce the myocardial stress to a larger extent, the surgical procedure might be preferential as opposed to the percutaneous procedure that is known to typically result in a higher level of residual RVOT difference. The current workflow, however, cannot provide the exact estimate of post-PVR physiology. The level of RVOT resistance decrease may depend on a number of factors (e.g. actual morphology of RVOTO, type of prosthetic valve used, initial type of repair). Possibly, patient-specific computational fluid dynamics simulations on the RVOT and PA could contribute to predict the decrease of the RV-to-PA pressure difference ${ }^{10,11,40}$. This is, however, out of scope of the current work.

The complete validation of the in silico predicted relationship between the postPVR contractility and RVOT resistance is limited due to the limitation of the study ${ }^{15}$, in which the only post-PVR measured pressures were available. In addition, the number of patients used in the present study is relatively small to draw universal conclusions regarding the response of RV mechanics to decreased RVOT pressure difference. Therefore, a detailed study with extended cohort of patients and simultaneous acquisition of pressure-volume data during the intervention (e.g. by a conductance catheter) is a natural follow-up to the present paper. The current modeling approach predicts an instantaneous change of ventricular mechanics at a given geometry in 
response to different levels of RVOTO whereas the long-term effect of PVR on the mechanical and morphological properties remain unknowable. In the future we propose to combine our prediction of immediate response to PVR with a kinematic growth theory framework ${ }^{12,14,21,23}$ or machine learning approach ${ }^{34}$, which could include some distinct features obtained from advanced image data analysis constrained by a biomechanical model ${ }^{6}$. In particular, it will be interesting to study the morphological change of the ventricular cavity to mechanical unloading at several points along the predicted (1D) line, where a given change of intraventricular pressure with respect to pre-PVR could be considered as a stress-based unloading stimuli ${ }^{25}$.

Biomechanical modeling has a potential to provide an insight into the evolution of RV mechanics. We have demonstrated the ability of the model to predict the mechanical response of the ventricle under progressive recovery of RV ESP through decreasing levels of RVOTO. Our results show a linear relationship between contractility and the level of RVOTO post-PVR. This step could contribute to the optimal clinical management for individual patients. The future objective would be to suggest what type of intervention (e.g. type or size of prosthesis, or percutaneous vs. surgical approach) would be the best for a given patient and combine with our ventricularmechanics prediction. In the current paper we demonstrated the approach on the cohort of tetralogy of Fallot patient with pulmonary valve pathology and right ventricular overload. This type of model-derived predicted outcome may have relevant application even in other valvular pathologies (e.g. aortic valve pathologies). 


\section{Conflict of interest statement:}

The authors declare that they have no competing interests.

\section{Acknowledgements:}

We would like to acknowledge Dr Philippe Moireau, Inria research team MEDISIM, for the development of the cardiac simulation software CardiacLab used in this work.

This work was supported by the Inria-UTSW Associated Team TOFMOD. It was also funded from by the W. B. \& Ellen Gordon Stuart Trust, The Communities Foundation of Texas and by the Pogue Family Distinguished Chair (award to Dr F. Gerald Greil in February, 2015). The work was in addition supported by the Ministry of Health of the Czech Republic [NV19-08-00071]. Research reported in this publication was supported by Children's Health ${ }^{S M}$, but the content is solely the responsibility of the authors and does not necessarily represent the official views of Children's Health ${ }^{\text {SM }}$. 


\section{References}

1. Arts, T., T. Delhaas, P. Bovendeerd, X. Verbeek, and F. W. Prinzen. Adaptation to mechanical load determines shape and properties of heart and circulation: the CircAdapt model. Am. J. Physiol.-Heart Circ. Physiol. 288:H1943-H1954, 2005.

2. Asner, L., M. Hadjicharalambous, R. Chabiniok, D. Peresutti, E. Sammut, J. Wong, G. Carr-White, P. Chowienczyk, J. Lee, A. King, N. Smith, R. Razavi, and D. Nordsletten. Estimation of passive and active properties in the human heart using 3D tagged MRI. Biomech. Model. Mechanobiol. 15:1121-1139, 2016.

3. Baumgartner, H., P. Bonhoeffer, N. M. S. De Groot, F. de Haan, J. E. Deanfield, N. Galie, M. A. Gatzoulis, C. Gohlke-Baerwolf, H. Kaemmerer, P. Kilner, F. Meijboom, B. J. M. Mulder, E. Oechslin, J. M. Oliver, A. Serraf, A. Szatmari, E. Thaulow, P. R. Vouhe, E. Walma, Task Force on the Management of Grown-up Congenital Heart Disease of the European Society of Cardiology (ESC), Association for European Paediatric Cardiology (AEPC), and ESC Committee for Practice Guidelines (CPG). ESC Guidelines for the management of grown-up congenital heart disease (new version 2010). Eur. Heart J. 31:2915-2957, 2010.

4. Bestel, J., F. Clément, and M. Sorine. A Biomechanical Model of Muscle Contraction. , 2001.doi:10.1007/3-540-45468-3_143

5. Caruel, M., R. Chabiniok, P. Moireau, Y. Lecarpentier, and D. Chapelle. Dimensional reductions of a cardiac model for effective validation and calibration. Biomech. Model. Mechanobiol. 13:897-914, 2014.

6. Castellanos, D. A., K. Škardová, A. Bhattaru, E. Berberoglu, G. Greil, A. Tandon, J. Dillenbeck, B. Burkhardt, T. Hussain, M. Genet, and R. Chabiniok. Left Ventricular Torsion Obtained Using Equilibrated Warping in Patients with Repaired Tetralogy of Fallot. Pediatr. Cardiol. 42:1275-1283, 2021. 
7. Chabiniok, R., P. Moireau, P.-F. Lesault, A. Rahmouni, J.-F. Deux, and D. Chapelle. Estimation of tissue contractility from cardiac cine-MRI using a biomechanical heart model. Biomech. Model. Mechanobiol. 11:609-630, 2012.

8. Chabiniok, R., V. Y. Wang, M. Hadjicharalambous, L. Asner, J. Lee, M. Sermesant, E. Kuhl, A. A. Young, P. Moireau, M. P. Nash, D. Chapelle, and D. A. Nordsletten. Multiphysics and multiscale modelling, data-model fusion and integration of organ physiology in the clinic: ventricular cardiac mechanics. Interface Focus 6:, 2016.

9. Chapelle, D., P. L. Tallec, P. Moireau, and M. Sorine. An energy-preserving muscle tissue model: formulation and compatible discretizations. Int. J. Multiscale Comput. Eng. 10:189, 2012.

10. Donati, F., S. Myerson, M. M. Bissell, N. P. Smith, S. Neubauer, M. J. Monaghan, D. A. Nordsletten, and P. Lamata. Beyond Bernoulli: Improving the Accuracy and Precision of Noninvasive Estimation of Peak Pressure Drops. Circ. Cardiovasc. Imaging 10:e005207, 2017.

11. Fučík, R., R. Galabov, P. Pauš, P. Eichler, J. Klinkovský, R. Straka, J. Tintěra, and R. Chabiniok. Investigation of phase-contrast magnetic resonance imaging underestimation of turbulent flow through the aortic valve phantom: Experimental and computational study using lattice Boltzmann method. Magn. Reson. Mater. Phys. Biol. Med. 33:649-662, 2020.

12. Genet, M., L. C. Lee, B. Baillargeon, J. M. Guccione, and E. Kuhl. Modeling Pathologies of Diastolic and Systolic Heart Failure. Ann. Biomed. Eng. 44:112-127, 2016.

13. Geva, T. Repaired tetralogy of Fallot: the roles of cardiovascular magnetic resonance in evaluating pathophysiology and for pulmonary valve replacement decision support. $J$. Cardiovasc. Magn. Reson. 13:9, 2011. 
14. Göktepe, S., O. J. Abilez, and E. Kuhl. A generic approach towards finite growth with examples of athlete's heart, cardiac dilation, and cardiac wall thickening. J. Mech. Phys. Solids 58:1661-1680, 2010.

15. Gusseva, M., T. Hussain, C. H. Friesen, P. Moireau, A. Tandon, C. Patte, M. Genet, K. Hasbani, G. Greil, D. Chapelle, and R. Chabiniok. Biomechanical Modeling to Inform Pulmonary Valve Replacement in Tetralogy of Fallot Patients after Complete Repair. Can. J. Cardiol. , 2021.doi:10.1016/j.cjca.2021.06.018

16. Hadjicharalambous, M., R. Chabiniok, L. Asner, E. Sammut, J. Wong, G. Carr-White, J. Lee, R. Razavi, N. Smith, and D. Nordsletten. Analysis of passive cardiac constitutive laws for parameter estimation using 3D tagged MRI. Biomech. Model. Mechanobiol. 14:807$828,2015$.

17. Hill, A. V. The heat of shortening and the dynamic constants of muscle. Proc. R. Soc. Lond. Ser. B - Biol. Sci. 126:136-195, 1938.

18. Holzapfel, G. A., and R. W. Ogden. Constitutive modelling of passive myocardium: a structurally based framework for material characterization. Philos. Trans. R. Soc. Math. Phys. Eng. Sci. 367:3445-3475, 2009.

19. Hunter, P. J., and B. H. Smaill. The analysis of cardiac function: A continuum approach. Prog. Biophys. Mol. Biol. 52:101-164, 1988.

20. Huxley, A. F. Muscle structure and theories of contraction. Prog. Biophys. Biophys. Chem. 7:255-318, 1957.

21. Kerckhoffs, R. C. P., J. H. Omens, and A. D. McCulloch. A single strain-based growth law predicts concentric and eccentric cardiac growth during pressure and volume overload. Mech. Res. Commun. 42:40-50, 2012.

22. Kimmig, F., D. Chapelle, and P. Moireau. Thermodynamic properties of muscle contraction models and associated discrete-time principles. Adv. Model. Simul. Eng. Sci. 6:6, 2019. 
23. Kroon, W., T. Delhaas, T. Arts, and P. Bovendeerd. Computational modeling of volumetric soft tissue growth: application to the cardiac left ventricle. Biomech. Model. Mechanobiol. 8:301-309, 2009.

24. Le Gall, A., F. Vallée, K. Pushparajah, T. Hussain, A. Mebazaa, D. Chapelle, É. Gayat, and R. Chabiniok. Monitoring of cardiovascular physiology augmented by a patient-specific biomechanical model during general anesthesia. A proof of concept study. PLOS ONE $15: \mathrm{e} 0232830,2020$.

25. Lee, L. C., M. Genet, G. Acevedo-Bolton, K. Ordovas, J. M. Guccione, and E. Kuhl. A computational model that predicts reverse growth in response to mechanical unloading. Biomech. Model. Mechanobiol. 14:217-229, 2015.

26. Lumens, J., and T. Delhaas. Cardiovascular Modeling in Pulmonary Arterial Hypertension: Focus on Mechanisms and Treatment of Right Heart Failure Using the CircAdapt Model. Am. J. Cardiol. 110:S39-S48, 2012.

27. Lumens, J., T. Delhaas, B. Kirn, and T. Arts. Three-Wall Segment (TriSeg) Model Describing Mechanics and Hemodynamics of Ventricular Interaction. Ann. Biomed. Eng. 37:2234-2255, 2009.

28. Lumens, J., S. Fan Chun-Po, J. Walmsley, D. Yim, C. Manlhiot, A. Dragulescu, L. GrosseWortmann, L. Mertens, W. Prinzen Frits, T. Delhaas, and K. Friedberg Mark. Relative Impact of Right Ventricular Electromechanical Dyssynchrony Versus Pulmonary Regurgitation on Right Ventricular Dysfunction and Exercise Intolerance in Patients After Repair of Tetralogy of Fallot. J. Am. Heart Assoc. 8:e010903, 2019.

29. Lurz P., Nordmeyer J., Muthurangu V., Khambadkone S., Derrick G., Yates R., Sury M., Bonhoeffer P., and Taylor A. M. Comparison of Bare Metal Stenting and Percutaneous Pulmonary Valve Implantation for Treatment of Right Ventricular Outflow Tract Obstruction. Circulation 119:2995-3001, 2009. 
30. McCulloch, A. D., B. H. Smaill, and P. J. Hunter. Left ventricular epicardial deformation in isolated arrested dog heart. Am. J. Physiol.-Heart Circ. Physiol. 252:H233-H241, 1987.

31. Niederer, S. A., G. Plank, P. Chinchapatnam, M. Ginks, P. Lamata, K. S. Rhode, C. A. Rinaldi, R. Razavi, and N. P. Smith. Length-dependent tension in the failing heart and the efficacy of cardiac resynchronization therapy. Cardiovasc. Res. 89:336-343, 2011.

32. Oosterhof, T., F. J. Meijboom, H. W. Vliegen, M. G. Hazekamp, A. H. Zwinderman, B. J. Bouma, A. P. J. van Dijk, and B. J. M. Mulder. Long-term follow-up of homograft function after pulmonary valve replacement in patients with tetralogy of Fallot. Eur. Heart $J$. 27:1478-1484, 2006.

33. Quail, M. A., A. Frigiola, A. Giardini, V. Muthurangu, M. Hughes, P. Lurz, S. Khambadkone, J. E. Deanfield, V. Tsang, and A. M. Taylor. Impact of pulmonary valve replacement in tetralogy of Fallot with pulmonary regurgitation: a comparison of intervention and nonintervention. Ann. Thorac. Surg. 94:1619-1626, 2012.

34. Regazzoni, F., D. Chapelle, and P. Moireau. Combining data assimilation and machine learning to build data-driven models for unknown long time dynamics-Applications in cardiovascular modeling. Int. J. Numer. Methods Biomed. Eng. 37:e3471, 2021.

35. Ruijsink, B., K. Zugaj, K. Pushparajah, and R. Chabiniok. Model-Based Indices of EarlyStage Cardiovascular Failure and Its Therapeutic Management in Fontan Patients. , 2019.doi:10.1007/978-3-030-21949-9_41

36. Ruijsink, B., K. Zugaj, J. Wong, K. Pushparajah, T. Hussain, P. Moireau, R. Razavi, D. Chapelle, and R. Chabiniok. Dobutamine stress testing in patients with Fontan circulation augmented by biomechanical modeling. PLOS ONE 15:e0229015, 2020.

37. Sainte-Marie, J., D. Chapelle, R. Cimrman, and M. Sorine. Modeling and estimation of the cardiac electromechanical activity. Comput. Struct. 84:1743-1759, 2006. 
38. Sermesant, M., R. Chabiniok, P. Chinchapatnam, T. Mansi, F. Billet, P. Moireau, J. M. Peyrat, K. Wong, J. Relan, K. Rhode, M. Ginks, P. Lambiase, H. Delingette, M. Sorine, C. A. Rinaldi, D. Chapelle, R. Razavi, and N. Ayache. Patient-specific electromechanical models of the heart for the prediction of pacing acute effects in CRT: A preliminary clinical validation. Med. Image Anal. 16:201-215, 2012.

39. Stergiopulos, N., B. E. Westerhof, and N. Westerhof. Total arterial inertance as the fourth element of the windkessel model. Am. J. Physiol. 276:H81-88, 1999.

40. Švihlová, H., J. Hron, J. Málek, K. R. Rajagopal, and K. Rajagopal. Determination of pressure data from velocity data with a view toward its application in cardiovascular mechanics. Part 1. Theoretical considerations. Int. J. Eng. Sci. 105:108-127, 2016.

41. Valente, A. M., K. Gauvreau, G. E. Assenza, S. V. Babu-Narayan, J. Schreier, M. A. Gatzoulis, M. Groenink, R. Inuzuka, P. J. Kilner, Z. Koyak, M. J. Landzberg, B. Mulder, A. J. Powell, R. Wald, and T. Geva. Contemporary predictors of death and sustained ventricular tachycardia in patients with repaired tetralogy of Fallot enrolled in the INDICATOR cohort. Heart 100:247-253, 2014.

42. Wang, V. Y., P. M. F. Nielsen, and M. P. Nash. Image-Based Predictive Modeling of Heart Mechanics. Annu. Rev. Biomed. Eng. 17:351-383, 2015. 


\section{Supplementary figures:}
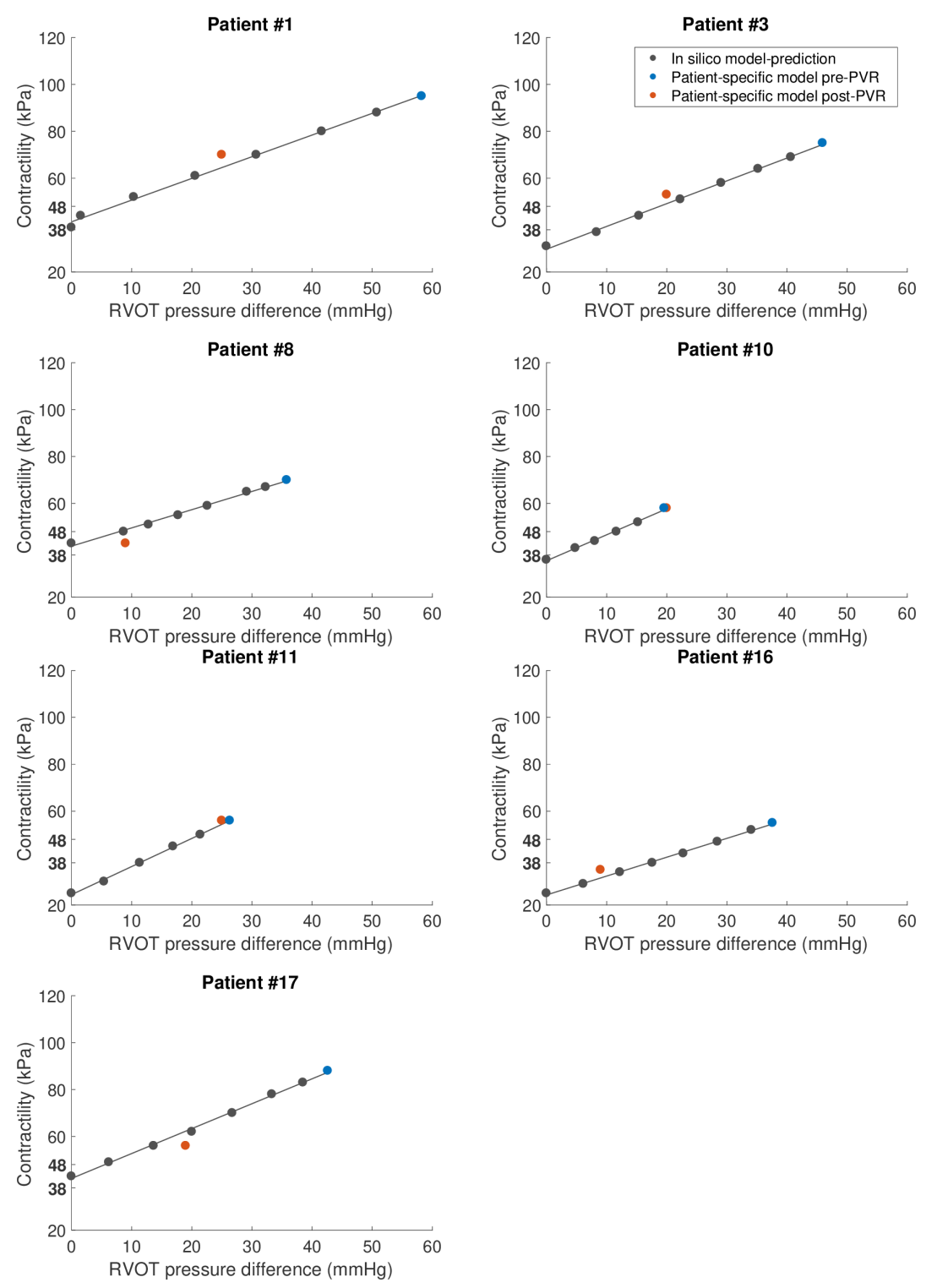

Supplementary Figure S1. In silico prediction of right ventricular (RV) con- tractility post-PVR (black points) for the patients from high right ventricular outflow tract obstruction (RVOTO) group. Filled blue and orange points are the contractilities in the pre- and post-PVR patient-specific models, respectively. 

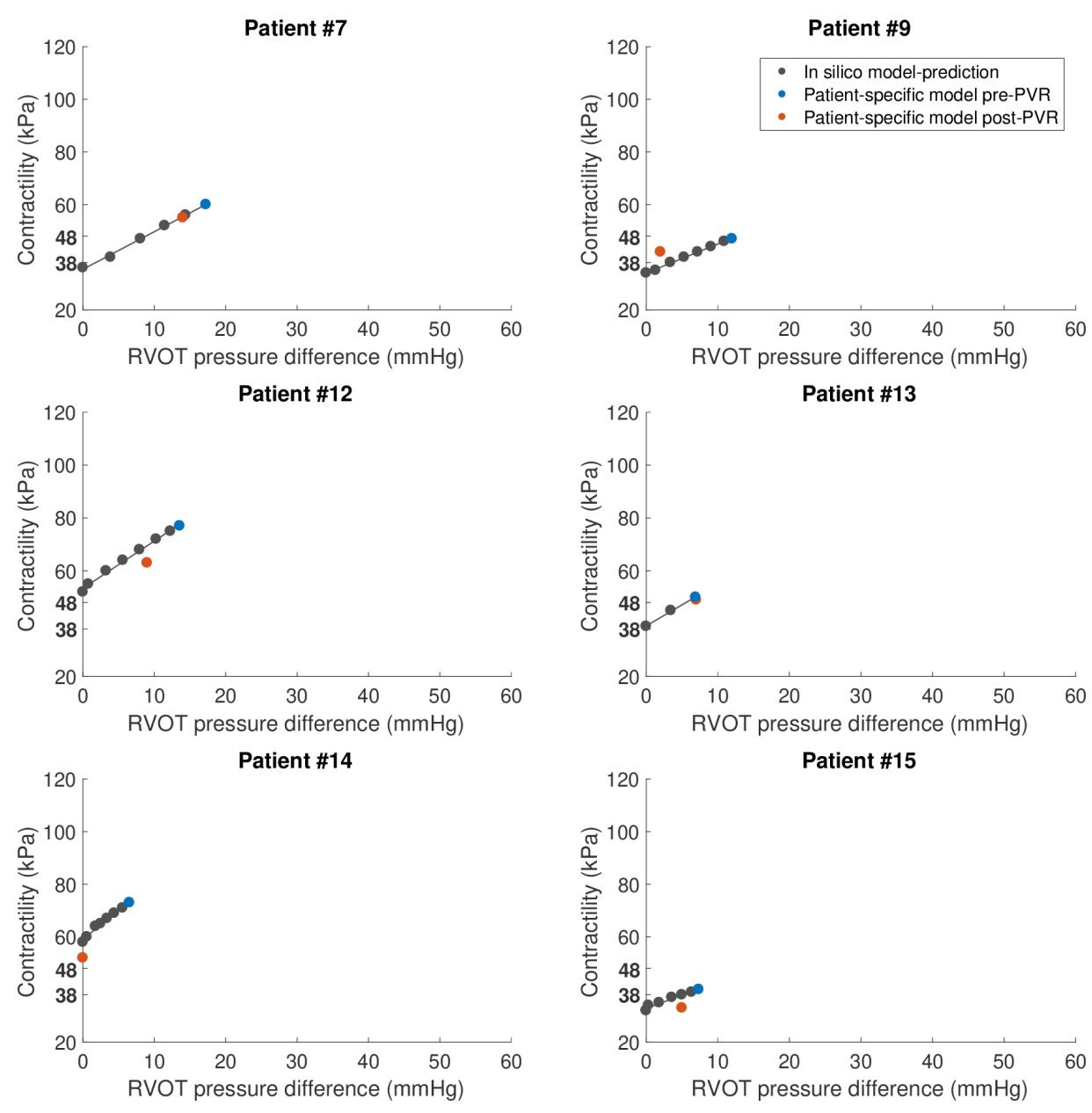

Supplementary Figure S2. In silico prediction of right ventricular (RV) con- tractility post-PVR (black points) for the patients from low right ventricular outflow tract obstruction (RVOTO) group. Filled blue and orange points are the contractilities in the pre- and post-PVR patient-specific models, respectively. 